\title{
The Effect of Form Factors and Control Types on Unsorted List Search for Full Touch Phone
}

\author{
Jongkee Lee ${ }^{1}$, Jaekyu Park ${ }^{1}$, Junyoung Kim ${ }^{1}$, Jaeho Choe ${ }^{2}$ Eui S. Jung ${ }^{1}$ \\ ${ }^{1}$ Department of Industrial Management Engineering, Korea University, Seoul, 136-701 \\ ${ }^{2}$ Department of Industrial Management Engineering, Daejin University, Pocheon, 487-711
}

\begin{abstract}
Objective: The aim of this paper is to inquire into the influences form factors and control types affect a search time and comfort at list menu of full touch phone. Background: Various studies have been proceeded that are related to the optimum touch area for enhancing usability of control and legibility in mobile touch device. In the environment of list menu which is widely used to provide various information effectively, however, not only comprehensive consideration for legibility and control is to be seek but also research for control type which is to scroll a list. Method: This study executed form factor experiment to inquire into the influence that font size, height of row and fixed area affect searching time and comfort in the while information processing even if the information on the list is unsorted in alphabetical order. Among the result of form factor experiment, control type experiment was executed by selecting shortest performance time, highest legibility comfort and control comfort. Control type experiment was implemented to figure out the influence which existing flicking type, scrolling bar type, newly established button page type and button raw types affect performance time and subjective comfort depending on location of the information. Results: Font size 12pt, height of row $7 \mathrm{~mm}$ and fixed area $15 \mathrm{~mm}$ was shortest performance time and got highest comfort and legibility score in form factor experiment. A Button page which was newly proposed type was shortest performance time and got highest comprehensive comfort in control type experiment. Conclusion: Form factor experiment showed similar results with the study through reading a long passage of character or controlling a grid icon type. However, height of row turned out to affect not only touch area for control but also legibility by ruling space between the lines. Button page type which was newly proposed showed shortest performance time and got highest comprehensive comfort. Because Button page type needs few finger movements than other control types and implements search in the fixed form, unlikely other type which list keeps moving. Application: This study should be applied in deciding form factors and control type for scroll when designing a list menu of full touch phone.
\end{abstract}

Keywords: Full touch phone, List menu, Form factor, Control type

\section{Introduction}

터치 기반의 휴대용 기기나 PDA에서 텍스트의 정보를 효율적으로 제공하는데 리스트 메뉴가 널리 사용되어 왔다
(Quinn and Cockburn, 2009). 최근 풀 터치 휴대폰에서도 다양한 텍스트의 정보를 비교적 작은 크기의 화면상에서 효 율적으로 제공하기 위하여 여러 메뉴방식 중 리스트 메뉴를 활용하고 있다(Lee and Lee, 2007). 대표적인 예로서 전화 번호부, 음악목록, 뉴스 등과 같은 어플리케이션에서 리스트

Corresponding Author: Eui S. Jung. Department of Industrial and Management Engineering, Korea University, Seoul, $136-701$.

Phone: 02-3290-3391, E-mail: ejung@korea.ac.kr

Copyright@2012 by Ergonomics Society of Korea(pISSN:1229-1684 eISSN:2093-8462). All right reserved.

(c) This is an open-access article distributed under the terms of the Creative Commons Attribution Non-Commercial License(http://creativecommons.org/licenses/by-nc/3.0/), which permits unrestricted non-commercial use, distribution, and reproduction in any medium, provided the original work is properly cited. 
메뉴가 주로 활용되고 있다. 이러한 리스트 메뉴는 전화번호 부나 음악목록 같이 한글 자음순서대로 정렬된 경우와 뉴스 나 영화목록 같이 시간순서대로 정렬된 경우로 나뉠 수 있다. 이러한 리스트 메뉴의 사용성은 글자 크기, 한 행의 높이, 스 크롤 방법, 시각화 기법 등의 다양한 요인들에 의하여 영향 을 받는다(Hwang, 2004). 하지만 텍스트의 정보를 효율적 으로 제공하는 방법에 관한 연구(Kim et al., 2004)나 터치 기반의 휴대용 기기에서 조작성을 높이기 위한 터치영역에 관한 연구(Park et al., 2006)는 장문을 읽는 환경이나 격자 형태의 메뉴환경에 초점을 맞추어 왔으며, 리스트 메뉴를 사 용하는 환경에서의 연구는 미흡한 실정이다.

터치 기반의 휴대용 기기에서 텍스트로 정보를 제공할 때 가독성은 사용성을 평가하는 중요한 요소가 되어 왔다. 가 독성은 텍스트가 얼마나 읽기 쉽고 이해하기 용이하게 제 공되어 있는지를 의미하며, 이전부터 VDT(Visual Display Terminal)에 이르기까지 한글 가독성과 관련된 다양한 연 구가 진행되어 왔다(Kong et al., 2010). 화면의 크기가 작 아 공간적 제약이 있는 휴대용 기기에서는 가독성에 영향을 미치는 다양한 요소가 존재한다(Lee et al., 2009). 글자의 특성에 관한 연구에서는 폰트의 종류와 크기(Kim et al., 2004), 자간간격과 행간간격 (Hwang et al., 1998) 등이 가 독성에 미치는 영향이 크다고 알려져 왔다. 글자의 특성 이 외에는 화면 크기와 밝기대비 등이 가독성에 영향을 미친다 (Lee and Kim, 2004). 따라서 이전 가독성과 관련된 연구 들은 주로 고정된 환경에서 텍스트의 가독성을 높일 수 있 는 방안에 초점을 맞추어 왔다. 본 연구에서는 리스트 메뉴 중 한글 자음순서대로 정렬되어 있지 않은 환경을 대상으로 하였다(Figure 1). 이는 한글자음 순서대로 정렬되어 있는 환경에서는 원하는 정보의 위치를 예측할 수 있어 텍스트를 모두 읽지 않고 예측되는 위치로 빠르게 이동할 수 있기 때 문이다. 따라서 리스트 메뉴를 스크롤 하면서 움직이는 정보 를 하나씩 탐색하는 환경에서 텍스트의 특성이 가독성에 미 치는 영향에 관하여 알아보았다.

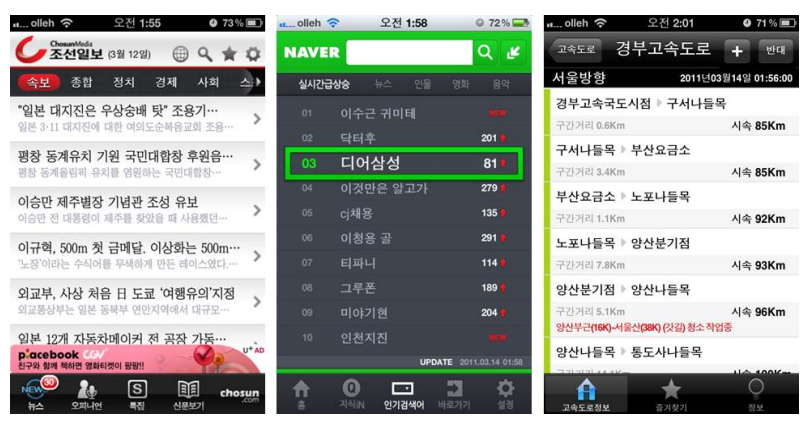

Figure 1. The example of unsorted list
터치 기반의 휴대용 기기는 디스플레이부와 조작부가 분 리되어 있는 버튼 방식과는 달리 화면상에서 직접적인 접촉 을 통해 입력하는 방식으로 좀 더 직관적인 인터페이스를 제 공하고 있다(Ebner et al., 2010). 하지만 화면의 공간적인 제약으로 인해 사용자 인터페이스(User Interface)를 설계 할 때 간섭을 줄이고 사용성을 높일 수 있는 최적의 터치영 역과 관련된 이슈가 부각되었고, 이에 따른 다양한 연구들이 진행되어 왔다(Parhi et al., 2005). 최적 터치영역의 크기는 디스플레이의 크기에 따라 달라질 수 있는데, 비교적 큰 사 이즈에서 숫자 키패드를 누를 때 적정 사이즈는 약 $20 \mathrm{~mm}$ (Colle and Hiszem, 2004), 비교적 작은 사이즈에서는 약 $7 \mathrm{~mm}$ 이상의 버튼 크기 (Park et al., 2006)에서 좋은 사용 성을 보였다. 또한 조작하는 도구에 따라 다른 수행도를 보 일 수 있는데, 약 $8 \mathrm{~mm}$ 이상의 버튼 크기를 사용할 때 손가 락으로 조작하는 것이 스타일러스팬으로 조작하는 것과 유 사한 입력시간과 에러율을 보였다(Hong et al., 2009). 최적 의 버튼 크기 이외에도 가로길이, 세로길이, 가로간격, 세로 간격은 사용자의 만족도에 영향을 미치는 설계변수로 활용 될 수 있다(Kim and Choi, 2007). 하지만 최적 터치영역과 관련된 연구들은 격자형태의 메뉴에서 아이콘을 선택하거나 정사각형의 숫자 키패드를 누르는 환경에서 이루어졌으며, 리스트 메뉴를 스크롤하여 직사각형의 한 행을 선택하는 리 스트 메뉴환경에서의 연구는 미흡한 실정이다. 따라서 본 연 구에서는 리스트 메뉴에서 터치영역을 한 행의 높이로 하여 기존 아이콘 형태의 터치영역과 비교하였다.

풀 터치 휴대폰에서 화면전환을 위한 조작방식은 여러 가 지가 있으며, 그 중 손가락을 이용한 플릭(Flick)이 가장 널 리 사용되고 있는 조작방식이다. Flick은 손가락으로 화면을 일정 방향으로 쓸면서 밀듯이 조작하면 페이지가 이동하거 나 리스트가 스크롤 되는 것을 말한다. Flick은 속도에 민감 하여 긴 리스트 메뉴를 스크롤할 때 페이지 이동속도와 멈 추는 속도를 잘 조절해서 설계해야 하며 (Kim, 2010), 손가 락을 움직이는 반복적 작업이 많아질 경우 사용자의 불편 도가 증가할 수 있다. 따라서 화면전환이나 스크롤 환경에 서 Flick을 넘어서려는 노력들이 계속되어 왔다(Quinn and Cockburn, 2009; Fitchett and Cockburn, 2009). 하지만 이전 연구들은 주로 화면의 이동, 확대, 축소와 같은 기능들 의 사용성을 높이기 위해 Flick과 같은 새로운 조작방식을 제안하는데 초점을 맞추어 왔으며, 리스트 스크롤과 같은 상 하의 연속적인 움직임에서 사용성을 높이기 위한 조작방식 이나 화면전환방식에 대한 연구는 미흡한 실정이다. 또한 리 스트 메뉴의 사용성에 미칠 수 있는 다양한 변수에 대한 종 합적인 고려가 부족하다. 따라서 본 연구에서는 리스트 메뉴 의 내용을 하나씩 읽어서 확인해야 하는 한글자음 순서대로 정렬되지 않은 환경에서 글자 크기에 대한 가독성의 차이를 
알아보았다. 또한 한 행의 높이에 대한 조작성의 차이와 스 크롤 영역에 대한 사용성과 만족도를 종합적으로 알아보고, 리스트 메뉴에서 적용될 수 있는 새로운 조작방식을 제안하 여 기존의 Flick 방식과 비교하였다.

\section{Form Factor Experiment}

본 실험에서는 풀 터치 휴대폰의 리스트 메뉴가 한글자음 순서대로 정렬되어 있지 않은 환경에서 사용자가 원하는 정 보를 찾고 선택할 때, 설계수치가 검색속도와 만족도에 미치 는 영향에 대하여 알아보았다. 피실험자는 풀 터치 휴대폰을 통하여 지정된 한글 단어를 찾는 Task를 수행하였다.

\subsection{Selection of the subjects}

본 실험에 참여한 피실험자는 풀 터치 휴대폰을 사용해본 경험이 있는 남자 6명, 여자 6명으로 구성된 20 30대 남녀 12 명을 대상으로 실시하였다. 평균연령은 26.6( \pm 2.9 ) 세이 며, 풀 터치 휴대폰 평균 사용기간은 $10.3( \pm 4.8)$ 개월이었 다. 이들은 모두 오른손잡이로 풀 터치 휴대폰을 조작하는데 지장이 없으며, 교정시력 1.0 이상의 피실험자를 대상으로 본 실험에 참여하도록 하였다.

\subsection{Experiment equipment}

본 실험을 위한 리스트 메뉴는 HTC사의 HD2에서 프로 그램을 통하여 구현되었다. HTC사의 HD2는 가로 $57 \mathrm{~mm}$, 세로 $94 \mathrm{~mm}$, 해상도 $480 \times 800$ 의 풀 터치 디스플레이를 탑 재한 휴대폰으로 Figure 2와 같이 피실험자가 왼손에 휴대 폰을 올려놓고 오른손 검지를 이용하여 조작하게 하였다. 리 스트 메뉴 내 정보는 3 자의 한글이름으로 중복과 정렬 없이 구성되었다.

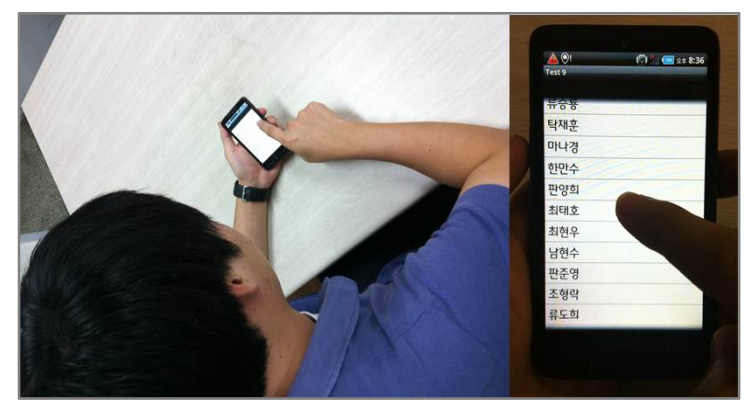

Figure 2. Form factor experiment environment

\subsection{Design of experiment}

터치 기반의 휴대용 기기에서 한글 가독성을 높일 수 있 는 글자 크기는 10pt이었으며 (Kim et al., 2004), 조작성을 높일 수 있는 버튼 크기는 $240 \times 320$ 픽셀을 갖는 장치에서 $8 \mathrm{~mm}$ 이상이었다(Hong et al., 2009). 따라서 본 연구에서 는 리스트 메뉴에서 원하는 정보를 찾아 선택하는데 있어 가독성과 조작성을 높일 수 있는 조합을 도출하기 위하여 글 자 크기(Font size), 한 행의 높이(Height of row)를 독립변 수로 선정하였다. 또한 현재 풀 터치 휴대폰의 리스트 메뉴 에서 네비게이션 버튼이나 검색 창이 상단에 위치하는 경우 가 많으며, 그 크기 또한 다양하게 존재한다. 이는 스크롤 시 리스트가 이동되지 않는 고정된 영역(Fixed area)으로 이에 따른 검색시간과 만족도의 영향력을 알아보기 위하여 이를 독립변수로 선정하였다.

종속변수는 사용자가 프로그램을 시작하여 원하는 정보를 선택하는데 까지 걸리는 시간을 프로그램 상에서 자동으로 측정하였고, 각 대안에 대하여 Task를 완료한 후 가독성 만 족도(Legibility Comfort) 와 조작성 만족도 (Control Comfort)를 100점 척도로 평가하였다(Table 1). 모든 실험 은 피실험자 개인의 특성에 따른 오차를 줄이고자 Within Subject Design으로 수행되었다. 실험대안의 제시 순서는 Balanced Latin Square 방법을 통하여 제시하였고, 학습효 과에 따른 오염을 최소화하기 위하여 각 대안마다 한글이름 의 순서를 랜덤하게 배치하였다. 따라서 모든 피실험자가 총 12 가지의 상이한 대안에 대하여 제시된 정보를 찾고, 가독 성 만족도와 조작성 만족도를 평가하였다.

Table 1. Variables definition of form factor experiment

\begin{tabular}{|c|c|c|c|}
\hline \multicolumn{2}{|c|}{ Variables } & Level & Description \\
\hline \multirow{3}{*}{ Independent } & Font size & 2 & $10,12(\mathrm{pt})$ \\
\hline & Height of row & 3 & $5,7,9(\mathrm{~mm})$ \\
\hline & Fixed area & 2 & $15,30(\mathrm{~mm})$ \\
\hline \multirow{2}{*}{ Dependent } & \multicolumn{3}{|c|}{ Completion time } \\
\hline & \multicolumn{3}{|c|}{ Legibility / Control comfort } \\
\hline
\end{tabular}

\subsection{Result}

측정 및 평가된 수행시간, 가독성 만족도, 조작성 만족도 에 대하여 각각 분산분석(ANOVA)을 실시하였고, 수행시 간에 대한 분산분석 결과는 Table 2 와 같다. 한 행의 높이 $(p-$ value $=0.013)$ 와 고정된 영역 $(p-$ value $=0.000)$ 에서 유의한 결과를 나타냈고, 글자 크기는 유의하지 않았다 
$(p$-value $=0.926)$. 각 변수간의 교호작용은 글자 크기와 한 행의 높이, 글자 크기와 고정된 영역에서 유의한 결과를 나타냈고, 나머지 교호작용은 유의하지 않았다.

Table 2. ANOVA results of completion time of form factor experiment

\begin{tabular}{c|c|c|c|c|c}
\hline & DF & SS & MS & $F$-value & $P$-value \\
\hline Font size & 1 & 0.042 & 0.042 & 0.009 & 0.926 \\
\hline Height of row & 2 & 12.221 & 6.111 & 5.302 & $0.013^{*}$ \\
\hline Fixed area & 1 & 55.081 & 55.081 & 49.265 & $0.000^{*}$ \\
\hline $\begin{array}{c}\text { Font size } \times \\
\text { Height of row }\end{array}$ & 2 & 15.594 & 7.797 & 3.6333 & $0.043^{*}$ \\
\hline $\begin{array}{c}\text { Font size } \times \\
\text { Fixed area }\end{array}$ & 1 & 14.364 & 14.364 & 12.440 & $0.004^{*}$ \\
\hline $\begin{array}{c}\text { Height of row } \times \\
\text { Fixed area }\end{array}$ & 2 & 10.726 & 5.363 & 2.421 & 0.112 \\
\hline $\begin{array}{c}\text { Font size } \times \\
\text { Height of row } \times \\
\text { Fixed area }\end{array}$ & 2 & 1.109 & 0.555 & 0.343 & 0.713 \\
\hline \multicolumn{5}{|c|}{$:$ Significant at $\alpha=0.05$}
\end{tabular}

수행시간에 대한 분산분석 결과 유의하였던 변수 중 한 행 의 높이가 수행시간에 미치는 영향을 알아보기 위하여 SNK (Student Newman-Keuls test)를 실시하였다. 그 결과 한 행의 높이 $5 \mathrm{~mm}$ 에서 6.62초, $7 \mathrm{~mm}$ 에서 6.75초로 A그룹, $9 \mathrm{~mm}$ 에서 7.29초로 B그룹으로 구분되었다(Figure 3). 이 는 한 행의 높이가 $9 \mathrm{~mm}$ 이상이 되면 사용자의 탐색시간이 증가한다는 것을 의미한다. 즉, 한 행의 높이는 한 화면에 표 시되는 행의 개수를 결정하여 스크롤하는 반복적 작업을 감 소 혹은 증가시키는 요인이 될 수 있기 때문에 $9 \mathrm{~mm}$ 미만의 설계가 수행시간을 줄일 수 있는 의미 있는 수치라는 것을

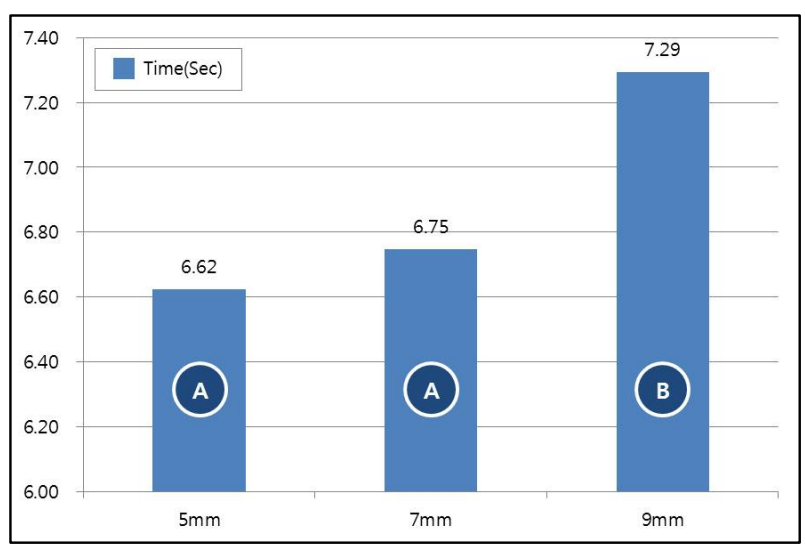

Figure 3. Height of row SNK results of completion time
나타낸다.

가독성 만족도에 대한 분산분석 결과, 글자 크기 ( $p$-value $=0.041)$ 와 한 행의 높이 $(p-$ value $=0.000)$ 에서 유의한 결 과가 나타났고, 고정된 영역 $(p-$ value $=0.384)$ 과 각 변수간 의 교호작용은 유의하지 않았다(Table 3). 이는 글자 크기 와 한 행의 높이가 리스트 메뉴에서 가독성에 영향을 미치 는 변수로 고려될 수 있다는 것을 의미한다.

Table 3. ANOVA results of legibility comfort of form factor experiment

\begin{tabular}{c|c|r|r|r|r}
\hline & DF & \multicolumn{1}{c|}{ SS } & \multicolumn{1}{c|}{ MS } & $F$-value & $P$-value \\
\hline Font size & 1 & 2062.674 & 2062.674 & 5.300 & $0.041^{*}$ \\
\hline Height of row & 2 & 23919.097 & 11959.549 & 30.558 & $0.000^{*}$ \\
\hline Fixed area & 1 & 62.674 & 62.674 & 0.821 & 0.384 \\
\hline $\begin{array}{c}\text { Font size } \times \\
\text { Height of row }\end{array}$ & 2 & 1160.764 & 580.382 & 2.553 & 0.100 \\
\hline $\begin{array}{c}\text { Font size } \times \\
\text { Fixed area }\end{array}$ & 1 & 8.507 & 8.507 & 0.162 & 0.694 \\
\hline $\begin{array}{c}\text { Height of row } \times \\
\text { Fixed area }\end{array}$ & 2 & 214.931 & 107.465 & 1.092 & 0.352 \\
\hline $\begin{array}{c}\text { Font size } \times \\
\text { Height of row } \times \\
\text { Fixed area }\end{array}$ & 2 & 114.931 & 57.465 & 1.118 & 0.334 \\
\hline \multicolumn{5}{|c|}{$:$ Significant at $\alpha=0.05$}
\end{tabular}

가독성 만족도에 대한 분산분석 결과 유의하였던 변수 중 한 행의 높이가 가독성 만족도에 미치는 영향력을 알아보기 위해 $\mathrm{SNK}$ 를 실시하였다. 그 결과 한 행의 높이는 $5 \mathrm{~mm}$ 가 $\mathrm{A}$ 그룹, $7 \mathrm{~mm}$ 와 $9 \mathrm{~mm}$ 가 B그룹으로 구분되었으며 가독성 만 족도 평균이 각각 41.46점, 66.67점, 70.52점이었다(Figure 4). 이는 한 행의 높이 $7 \mathrm{~mm}$ 이상이 가독성 만족도를 높일

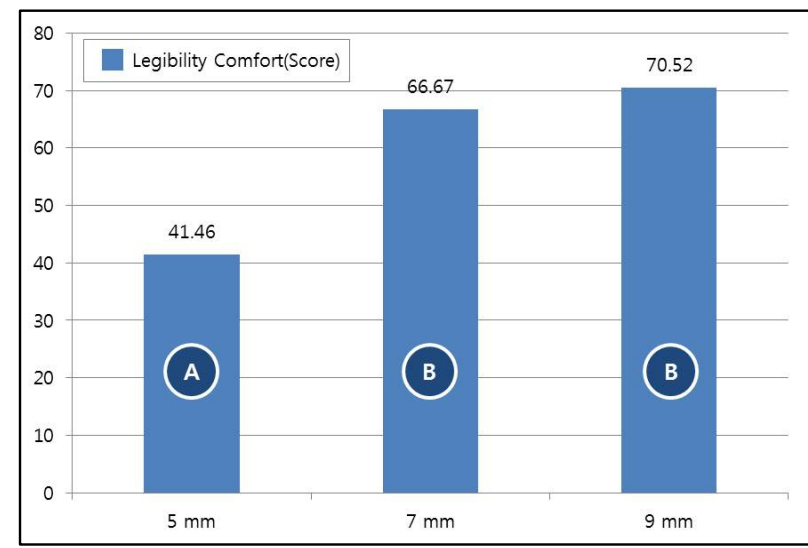

Figure 4. Height of row SNK results of legibility comfort 
수 있다는 것을 의미한다.

조작성 만족도에 대한 분산분석 결과, 한 행의 높이 $(p-$ value $=0.000)$ 와 고정된 영역 $(p-$ value $=0.008)$ 에서 유의 한 결과가 나타났고, 글자 크기 $(p-v a l u e=0.112)$ 와 각 변 수간의 교호작용은 유의하지 않았다(Table 4). 이는 한 행 의 높이와 고정된 영역이 리스트 메뉴에서 조작성에 영향을 미치는 변수로 고려될 수 있다는 것을 의미한다.

Table 4. ANOVA results of control comfort of form factor experiment

\begin{tabular}{c|c|r|r|r|c}
\hline & DF & \multicolumn{1}{c|}{ SS } & \multicolumn{1}{c|}{ MS } & $F$-value & $P$-value \\
\hline Font size & 1 & 756.250 & 756.250 & 2.978 & 0.112 \\
\hline Height of row & 2 & 25437.847 & 12718.924 & 34.282 & $0.000^{*}$ \\
\hline Fixed area & 1 & 336.111 & 336.111 & 10.160 & $0.008^{*}$ \\
\hline $\begin{array}{c}\text { Font size } \times \\
\text { Height of row }\end{array}$ & 2 & 688.542 & 344.271 & 2.515 & 0.103 \\
\hline $\begin{array}{c}\text { Font size } \times \\
\text { Fixed area }\end{array}$ & 1 & 6.250 & 6.250 & 0.086 & 0.775 \\
\hline $\begin{array}{c}\text { Height of row } \times \\
\text { Fixed area }\end{array}$ & 2 & 381.597 & 190.799 & 2.132 & 0.142 \\
\hline $\begin{array}{c}\text { Font size } \times \\
\text { Height of row } \times \\
\text { Fixed area }\end{array}$ & 2 & 94.792 & 47.396 & 0.643 & 0.535 \\
\hline
\end{tabular}

${ }^{*}:$ Significant at $\alpha=0.05$

조작성 만족도에 대한 분산분석 결과 유의하였던 변수 중 한 행의 높이가 조작성 만족도에 미치는 영향력을 알아보기 위해 $\mathrm{SNK}$ 를 실시하였다. 그 결과 한 행의 높이는 $5 \mathrm{~mm}$ 가 $\mathrm{A}$ 그룹으로, $7 \mathrm{~mm}$ 와 $9 \mathrm{~mm}$ 가 $\mathrm{B}$ 그룹으로 구분되었으며 조작 성 만족도의 각 평균은 $5 \mathrm{~mm}$ (42.81점), $7 \mathrm{~mm}$ (69.38점), $9 \mathrm{~mm}$ (72.40점)이었다(Figure 5). 이는 한 행의 높이 $7 \mathrm{~mm}$

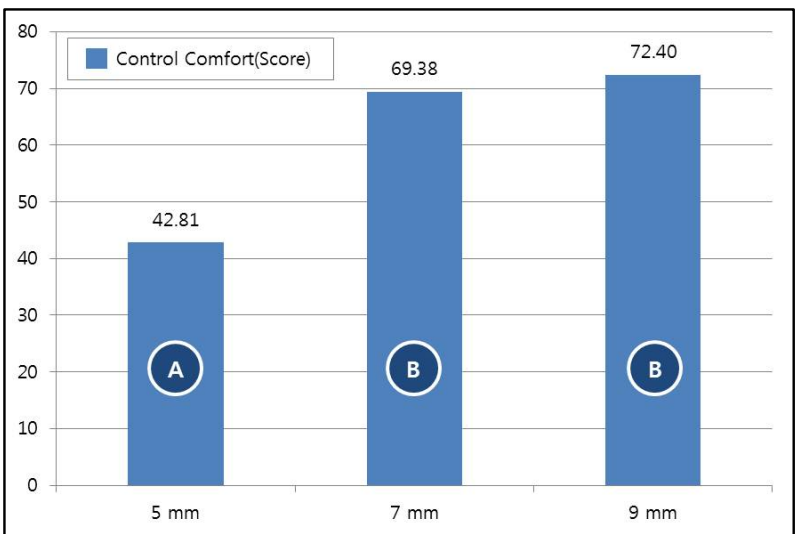

Figure 5. Height of row SNK results of control comfort
이상이 조작성 만족도를 높일 수 있다는 것을 의미한다.

종합해보면, 글자 크기의 변화는 가독성 만족도에 영향을 미치며 $12 \mathrm{pt}$ 가 가장 우수한 가독성 만족도를 나타냈다. 하 지만, 글자 크기가 원하는 정보를 찾기 위한 수행시간이나 조작성 만족도에 미치는 영향은 유의하지 않은 것으로 나타 났다. 한 행의 높이는 수행시간, 가독성 만족도, 조작성 만족 도 모두에 영향을 미치는 것으로 나타났으며, $7 \mathrm{~mm}$ 가 가장 우수한 결과를 나타냈다. 이는 한 행의 높이가 작으면 글자 의 행간간격이 좁아져 가독성 만족도를 낮추고, 한 행의 높 이가 넓으면 스크롤 작업이 반복되어 수행시간과 조작 만족 도에 부정적 영향을 미치는 것을 의미한다. 고정된 영역의 변화는 수행시간과 조작성 만족도에 영향을 미치는 것으로 나타났다. 이는 스크롤되는 영역을 결정하는 고정된 영역이 좁을수록 한번에 많은 행을 표시하고 스크롤할 수 있기 때 문에 수행시간이 짧고 조작성 만족도가 높은 것을 의미한다. 결론적으로, 수행시간을 줄이고 가독성 만족도와 조작성 만 족도를 높일 수 있는 대안으로 글자 크기 $12 \mathrm{pt}$, 한 행의 높 이 $7 \mathrm{~mm}$, 고정된 영역 $15 \mathrm{~mm}$ 가 선정되었다(Table 5).

Table 5. Total result of form factor experiment

\begin{tabular}{c|c|c|c}
\hline & $\begin{array}{c}\text { Completion } \\
\text { time }\end{array}$ & $\begin{array}{c}\text { Legibility } \\
\text { comfort }\end{array}$ & $\begin{array}{c}\text { Control } \\
\text { comfort }\end{array}$ \\
\hline Font size & $10 \mathrm{pt} \mathrm{or} 12 \mathrm{pt}$ & $12 \mathrm{pt}$ & $10 \mathrm{pt} \mathrm{or} 12 \mathrm{pt}$ \\
\hline Height of row & $7 \mathrm{~mm}$ & $7 \mathrm{~mm}$ & $7 \mathrm{~mm}$ \\
\hline Fixed area & $15 \mathrm{~mm}$ & $15 \mathrm{~mm}$ or $30 \mathrm{~mm}$ & $15 \mathrm{~mm}$ \\
\hline
\end{tabular}

\section{Control Type Experiment}

본 실험에서는 설계수치 실험에서 선정된 가독성 만족도 와 조작성 만족도를 높일 수 있는 대안의 조합으로 글자 크 기, 한 행의 높이, 고정된 영역을 고정하고 리스트 메뉴가 한 글자음 순서대로 정렬되어 있지 않은 환경에서 조작방식과 원하는 정보의 위치가 검색시간과 만족도에 미치는 영향에 대하여 알아보았다. 피실험자는 풀 터치 휴대폰을 통하여 지 정된 한글 문장을 찾는 Task를 수행하였다.

\subsection{Selection of the subjects}

본 실험에 참여한 피실험자는 풀 터치 휴대폰을 사용해본 경험이 있는 남자 12 명, 여자 12 명으로 구성된 20 30대 남녀 24명을 대상으로 실시하였다. 평균연령은 27.2( \pm 1.8 ) 세이며, 풀 터치 휴대폰 평균 사용기간은 11.9 ( \pm 3.5$)$ 개월 
이었다. 이들은 모두 오른손잡이로 풀 터치 휴대폰을 조작 하는데 지장이 없으며, 교정시력 1.0 이상의 피실험자를 대 상으로 본 실험에 참여하도록 하였다.

\subsection{Experiment equipment}

본 실험에서는 설계수치 실험에서 사용된 기기와 동일한 $\mathrm{HTC}$ 사의 HD2가 사용되었다. 피실험자는 왼손에 휴대폰을 올려놓고 오른손 검지를 이용하여 휴대폰을 조작하게 하였으 며, 리스트 메뉴 내 정보는 신문기사의 헤드라인으로 중복과 정렬 없이 구성되었다.

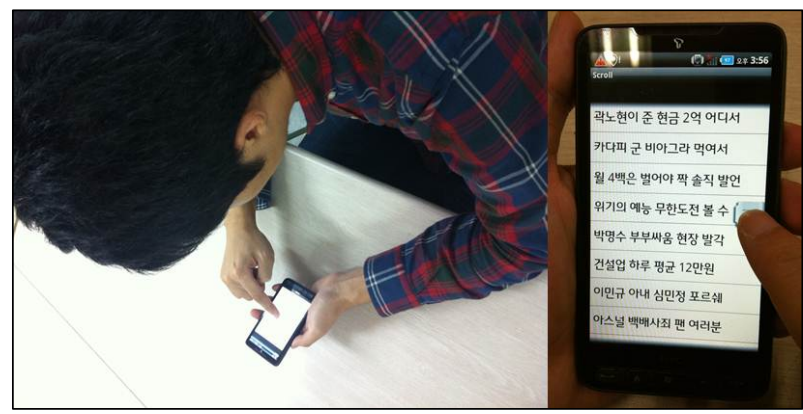

Figure 6. Control type experiment environment

\subsection{Design of experiment}

본 실험에서는 리스트 메뉴에서 원하는 정보를 찾기 위해 스크롤 하는 방법이 검색속도와 만족도에 미치는 영향에 대 하여 알아보기 위해 조작방식을 독립변수로 선정하였다. 조 작방식은 기존에 풀 터치 휴대폰의 리스트 메뉴에서 사용되 고 있는 조작방식인 Flick과 Scroll bar를 선정하였다. 하지 만 Flick과 Scroll bar는 화면의 전환속도가 빠르고 민감하 여 사용자가 속도를 조절하기 어렵고, 리스트가 길어질 경우 반복적인 손가락 움직임이 발생한다는 단점이 있다. 따라서 이러한 단점을 보안하면서 리스트 메뉴에 적용될 수 있는 새 로운 조작방식으로 Button page와 Button raw를 제안하였 다. Button page는 리스트 메뉴를 스크롤 할 수 있는 별도 의 버튼을 리스트 우측 상단과 하단에 위치시켜 한번의 버 튼입력으로 화면 전체를 전환시킬 수 있는 방식이다. 이는 원하는 정보가 리스트의 뒤에 위치하여도 Flick이나 Scroll bar와 같이 반복적인 손가락 움직임이 적다는 장점이 있다. Button raw는 Button page와 같은 위치에 버튼을 위치시켜 연속적인 버튼입력으로 한 행씩 연속적으로 이동하는 방식 이다. 이는 리스트의 스크롤 정도를 버튼입력여부로 조절할 수 있기 때문에 속도의 조절이 용이하다는 장점이 있다. 따
라서 조작방식은 4수준으로 Flick, Scroll bar, Button page, Button raw가 선정되었다(Figure 7). 또한 리스트에서 원하 는 정보의 위치가 검색속도와 만족도에 미치는 영향에 대하 여 알아보기 위해 정보의 위치를 독립변수로 선정하였다. 원 하는 정보의 위치는 3수준으로 총 152 개의 행으로 이루어

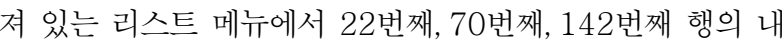
용으로 하여 각각을 Short, Middle, Long이라 명명하였다 (Table 6)

종속변수는 사용자가 프로그램을 시작하여 원하는 정보를 선택하는데 까지 걸리는 시간을 프로그램 상에서 자동으로 측정하게 하였고, 각 대안에 대한 Task를 완료한 후 100점 척도로 종합만족도(Total Comfort)를 평가하였다. 모든 실 험은 피실험자 개인의 특성에 따른 오차를 줄이고자 Within Subject Design으로 수행되었다. 실험대안의 제시 순서는 학습효과에 따른 오염을 최소화하기 위하여 Balanced Latin Square 방법을 통하여 제시하였다. 또한 4가지 조작방식에 대하여 미리 선별된 Task를 수행하면서 사용자가 기존 및 새로운 방식에 대해 적응할 수 있게 하였고, 이는 분석 데이 터에서 제외하였다. 따라서 모든 피실험자는 총 12 가지의 상이한 대안에 대하여 제시된 정보를 찾고, 종합만족도를 평 가하였다

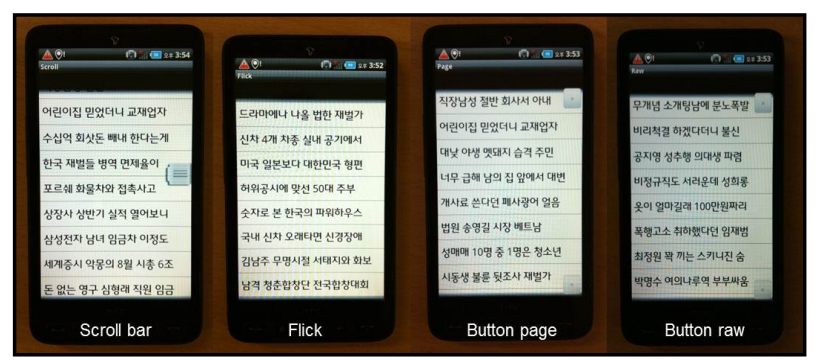

Figure 7. Control types

Table 6. Variables definition of control type experiment

\begin{tabular}{c|c|c|c}
\hline \multicolumn{2}{c|}{ Variables } & Level & Description \\
\hline \multirow{2}{*}{ Independent } & $\begin{array}{c}\text { Control } \\
\text { type }\end{array}$ & 4 & $\begin{array}{c}\text { Flick, Scroll bar } \\
\text { Button page, Button raw }\end{array}$ \\
\cline { 2 - 4 } & $\begin{array}{c}\text { Target } \\
\text { location }\end{array}$ & 3 & Short, Middle, Long \\
\hline \multirow{2}{*}{ Dependent } & \multicolumn{3}{|c}{ Completion time } \\
\cline { 2 - 4 } & \multicolumn{3}{|c}{ Total comfort } \\
\hline
\end{tabular}

\subsection{Result}

측정 및 평가된 수행시간과 종합만족도에 대하여 분산분 
석을 실시하였다. 수행시간의 분산분석 결과 조작방식 $(p-$ value $=0.000)$, 원하는 정보의 위치 $(p-$ value $=0.000)$, 조 작방식과 원하는 정보의 위치의 교호작용 $(p-$ value $=0.003)$ 이 유의하였다(Table 7). 조작방식의 수행시간 평균은 Button page, Flick, Scroll bar, Button raw 순으로 빠르 게 나타났으며, 원하는 정보의 위치에 따른 수행시간 평균은 Short, Middle, Long 순으로 빠르게 나타났다.

Table 7. ANOVA results of completion time (Control type experiment)

\begin{tabular}{c|c|c|c|c|c}
\hline & DF & \multicolumn{1}{c|}{ SS } & MS & $F$-value & $P$-value \\
\hline Control type & 3 & 2003.412 & 667.714 & 14.007 & $0.000^{*}$ \\
\hline Target location & 2 & 270609.682 & 13804.841 & 484.634 & $0.000^{*}$ \\
\hline $\begin{array}{l}\text { Control type } \times \\
\text { Target location }\end{array}$ & 6 & 536.682 & 89.447 & 3.476 & $0.003^{*}$ \\
\hline \multicolumn{7}{c}{ * Significant at $\alpha=0.05$}
\end{tabular}

원하는 정보의 위치에 따라 조작방식이 수행시간에 미치 는 영향은 각각 다르게 나타났다(Figure 8). 원하는 정보의 위치가 Short인 경우에는 Button Page와 Flick, Button raw와 Scroll bar에서 각각 유사한 수행시간으로 보였으며, Middle인 경우에는 Flick, Button Raw, Scroll bar가 유사 한 수행시간을 보였고, Button page가 가장 빠른 수행시간을 보였다. Long인 경우에도 Middle과 유사한 경향을 보였으나 Button page, Flick, Button raw, Scroll bar 순으로 그 차이 가 Middle인 경우보다 더욱 커지는 경향이 나타났다. 이는 원하는 정보가 뒤에 위치할수록 리스트를 스크롤하는 조작 방식에 따라 수행시간이 달라진다는 것을 의미한다.

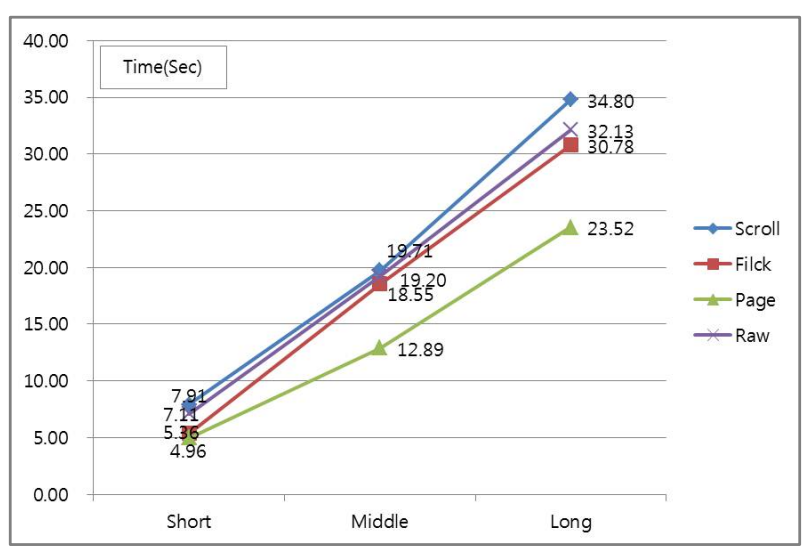

Figure 8. Interaction line of completion time

종합만족도에 대한 분산분석 결과 조작방식 ( $p$-value=
$0.000)$ 과 원하는 정보의 위치 $(p-$ value $=0.000)$ 에서 유의 한 차이를 보였으며, 교호작용은 유의하지 않았다(Table 8). 조작방식에 따른 종합만족도의 평균점수는 Button page, Flick, Button raw, Scroll bar 순으로 높은 것으로 나타났 으며, 원하는 정보의 위치에 따른 종합만족도의 평균점수는 Short, Middle, Long 순으로 높은 것으로 나타났다.

Table 8. ANOVA results of total comfort (Control type experiment)

\begin{tabular}{c|c|r|c|c|c}
\hline & DF & \multicolumn{1}{c|}{ SS } & MS & $F$-value & $P$-value \\
\hline Control type & 3 & 23187.153 & 7729.051 & 26.395 & $0.000^{*}$ \\
\hline Target location & 2 & 9925.000 & 4962.500 & 63.557 & $0.000^{*}$ \\
\hline $\begin{array}{c}\text { Control type } \times \\
\text { Target location }\end{array}$ & 6 & 424.306 & 70.718 & 1.695 & 0.126 \\
\hline \multicolumn{5}{c}{$:$ Significant at $\alpha=0.05$}
\end{tabular}

종합만족도에 대한 분산분석 결과 유의하였던 조작방식과 원하는 정보의 위치가 종합만족도에 미치는 영향을 알아보 기 위해 $\mathrm{SNK}$ 를 실시하였다. 그 결과 Scroll bar가 A그룹, Flick과 Button raw가 B그룹, Button page가 C그룹으로 분류되었다. 각 그룹간의 종합만족도 평균은 $\mathrm{C}, \mathrm{B}, \mathrm{A}$ 순으로 높은 것으로 나타났다(Figure 9). 이는 수행시간의 분산분석 결과와 유사한 경향을 나타냈으며, 리스트 메뉴를 스크롤할 때 Button page 방식이 종합만족도를 높일 수 있는 대안이 라는 것을 의미한다.

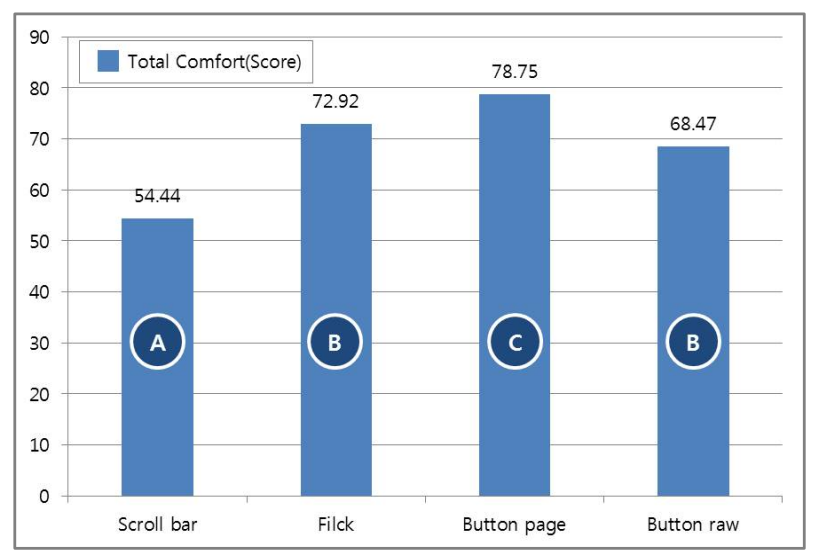

Figure 9. Control type SNK results of total comfort 


\section{Conclusion}

\subsection{Form factor}

본 연구는 한글자음 순서대로 정렬되어 있지 않은 리스트 메뉴에서 설계수치와 조작방식이 수행시간과 만족도에 미치 는 영향에 대하여 분석하였다. 설계수치는 글자 크기 $12 \mathrm{pt}$, 한 행의 높이 $7 \mathrm{~mm}$, 고정된 영역 $15 \mathrm{~mm}$ 에서 가장 우수한 수행시간과 가독성 및 조작성 만족도를 나타냈다. 이는 기존 의 장문을 읽는 환경이나 격자형태의 메뉴환경에서 진행되 었던 연구들과 유사한 결과를 나타냈다.

가독성 만족도에 대한 분산분석 결과, 글자의 특성이 가 독성에 미치는 영향에 관한 연구 중 글자 크기에 관한 연구 (Kim et al., 2004) 와 행간간격에 관한 연구(Hwang et al., 1998)와 유사한 결과를 나타냈으며, 이는 한 행의 높이가 조작을 위한 터치영역뿐만 아니라 가독성에 영향을 미치는 행간간격을 결정한다는 것을 의미한다. 하지만, 고정된 영역 의 차이로 인한 한 화면에 표시되는 행의 개수의 차이는 한 행의 정보를 읽는 가독성 만족도에 미치는 영향은 미비한 것 으로 나타났다. 조작성 만족도에 대한 분산분석 결과, 최적 터치영역에 관한 연구(Park et al., 2006)와 유사한 결과를 나타냈으며, 이는 한 행의 높이가 $7 \mathrm{~mm}$ 이상에서 조작성 만 족도를 보장할 수 있다는 것을 의미한다. 즉, 리스트 환경에 서 $7 \mathrm{~mm}$ 이상의 한 행의 높이는 터치를 위한 조작성뿐만 아 니라 리스트 스크롤을 위한 조작성을 모두 만족하는 수치임 을 의미한다. 또한 리스트 메뉴에서 검색 창이나 네비게이션 버튼과 같은 메뉴들이 포함된 고정된 영역의 크기는 리스트 스크롤 시 조작성에 영향을 미친다고 할 수 있다. 따라서 검 색시간과 가독성 및 조작성 만족도를 고려하여 리스트 메뉴 의 설계수치를 결정할 때 글자 크기, 한 행의 높이, 고정된 영역은 의미 있는 변수로 활용될 수 있을 것이다.

\subsection{Control type}

조작방식은 Button page 방식이 가장 우수한 수행시간과 종합만족도를 나타냈다. 수행시간에서는 Button page, Flick, Button raw, Scroll bar 순으로 빠르게 나타났으며, 원하는 정보가 뒤에 위치할수록 Button page 방식이 다른 방식들 에 비해 빠르게 나타났다. 이는 원하는 정보가 가까운 곳에 위치할 경우 리스트 메뉴의 화면전환 횟수가 적어 반복적인 손가락 움직임이나 스크롤 민감도를 조절하는 어려움이 적 다는 것을 의미한다. 하지만 원하는 정보가 뒤에 위치할 수 록 반복적인 손가락 움직임과 스크롤 민감도를 조절하는 어 려움이 증가하여 Flick, Button raw, Scroll bar 방식의 수행 시간이 증가한 것으로 판단된다. 하지만 Button page 방식
은 한 번의 조작으로 리스트가 페이지단위로 이동하기 때문 에 원하는 정보의 위치가 뒤에 위치하여도 반복적인 손가락 움직임이 크게 증가하지 않아 수행시간이 가장 짧게 나타 났다.

종합만족도에서도 수행시간과 동일하게 Button page, Flick, Button raw, Scroll bar 순으로 만족도가 높은 것으로 나타났다. 이는 Button page 방식의 한번의 조작에 따른 고 정된 정보에 대한 탐색이 Scroll bar, Flick, Button raw 방 식의 연속적인 조작에 따른 움직이는 정보에 대한 탐색보다 종합만족도에서 우수한 평가를 받은 것을 의미한다. Scroll bar 방식은 연속적인 조작과 움직이는 정보에 대한 탐색이 라는 점에서 Flick, Button raw 방식과 유사하지만 Scroll bar를 통한 스크롤은 민감도를 조절하기 어렵다는 점에서 가장 낮은 평가를 받은 것으로 판단된다. Button raw 방식 은 스크롤 민감도를 조절하는 측면에서 Scroll bar보다 우수 하나, 원하는 정보의 위치가 뒤에 위치할수록 민감도를 조절 하기 위하여 반복적인 조작이 필요하다. 따라서 Scroll bar 방식보다는 우수하지만 Flick 방식과 유사한 평가를 받은 것 으로 판단된다.

본 연구 결과, 리스트 메뉴의 스크롤 시 짧은 검색시간과 높은 사용자 만족도를 보장하기 위한 조작방식으로 Button page 방식이 선정되었다. 하지만 Button page 방식은 물리 적으로 Button이 위치하여야 하는 공간이 필요하며, 이는 화면상에서 다양한 정보를 제공하는데 제약이 될 수 있다. 따라서 가장 익숙한 조작방식인 Flick과 우수하게 평가된 화면 전환방식인 Button page의 장점을 결합한 새로운 조작 방식에 대한 연구가 필요할 것이다. 또한 본 연구는 비교적 작은 크기의 터치 디스플레이를 탑재한 풀 터치 휴대폰 환경 에서 이루어졌으며, Tablet PC와 같이 크기가 큰 터치 디스 플레이 환경에서의 연구가 추가적으로 필요할 것이다. 본 연 구가 향후 풀 터치 휴대폰에서 리스트 메뉴를 설계할 때 사 용성을 높일 수 있는 가이드라인으로 활용되기를 기대한다.

\section{References}

Colle, H. and Hiszem, K., Effect of key size and spacing on touch screen numeric keypad performance and user preference, Journal of Ergonomics, 47(13), 1406-1423, 2004.

Ebner, M., et al. "iPhone/iPad Human Interface Design", Proceedings of 6th Symposium of the WG HCI \& UE of the Austrian Computer Society, (pp. 489-492), Klagenfurt, Australia, 2010.

Fitchett, S. and Cockburn, A., MultiScroll: Using Multitouch Input to Disambiguate Relative and Absolute Mobile Scroll Modes, Proceedings of the 21th Australasian conference on Computer-Human Interaction, 
(pp. 23-27), Melbourne, Australia, 2009.

Hwang, K. M., Information Visualization of Menu Design on PDA Using Fisheye View, Master's thesis of Industrial Management Engineering of Korea University Graduate School, 2004.

Hwang, W. S., Bu, J. H. and Lee, D. C., "The Optimal Letter Spacing and Line Spacing of Korean on the Visual Display", Spring Conference on Ergonomics Society of Korea, 1(pp. 161-166), Jeju, 1998.

Hong, S. G., Park, J. C. and Kim, S. S., "A Study on Button Size and Spacing on Touch Screen PDA", Spring Conference on Ergonomics Society of Korea, (pp. 469-473), Daegu, 2009.

Kim, B. G., Kim, I. S. and Choi, J. H., "Readability Evaluation of Hangul Font with a Handheld Computer", Fall Conference on Ergonomics Society of Korea, Daechun, 2004.

Kim, J. and Choi, K. S., A User Satisfaction Based Touch Button Design, Applied Korean Institute of Industrial Engineers, 20(4), 539-546, 2007.

Kong, Y. K., et al. Subjective Assessments and Analysis of Letter Sizes Under Black and White Contrasts for Various Font Sizes Through the Irradiation-effect, Journal of the Ergonomics Society of Korea, 29(1), 107-112, 2010.

Kim, H., Guidelines for Satisfactory Flick Performances in Touch Screen Mobile Phone, Journal of the Ergonomics Society of Korea, 29(4), 541-546, 2010

Lee, S. E. and Lee, G. H., "K-Menu: A Keyword-based Dynamic Menu Interface for Small Computers", Proceedings of Computer/Human Interaction Conference, (pp. 2543-2548), San Jose, California, USA, 2007.

Lee, I. S., et al. Evaluation of Main Factors Affecting on the Legibility of One-Syllable Korean Characters and Numbers, Journal of the Ergonomics Society of Korea, 28(4), 1-7, 2009.

Lee, S. J. and Kim, J. W., "The Effect of Luminance Contrast \& Screen Size on Reading Performance in VDT Environment", Conference on Human Computer Interaction Society of Korea, Gangwon, 2004.

Park, J. H., et al. "Touch Key Design and Movement Models for Onehanded Thumb Input", Fall Conference on Ergonomics Society of Korea, Daejeon, 2006.

Parhi, P., et al. "Target Size Study for One-Handed Thumb Use on Small Touchscreen Devices", Proceedings of 8th International Conference on Human Computer Interaction with Mobile Devices and Services, (pp. 203-210), Espoo, Finland, 2006.

Quinn, P. and Cockburn, A., "Zoofing List Selections with Pressure-ZoomFlick-Scrolling", Proceedings of the 21st Annual Conference of the Australian Computer-Human Interaction Special Interest Group, (pp. 185-192), Melbourne, Australia, 2009.

\section{Author listings}

Jongkee Lee: 1jk1557@gmail.com

Highest degree: BS, Computer Science and Engineering, Hankuk University of Foreign Studies

Position title: MS. Candidate, Department of Industrial Management Engineering, Korea University

Areas of interest: Product Development, UX, UI

Jaekyu Park: ergopark@gmail.com

Highest degree: MS, Department of Industrial System and Information Engineering, Korea University

Position title: $\mathrm{PhD}$. Candidate, Department of Industrial Management Engineering, Korea University

Areas of interest: Product Development, Human Error, Workload

Junyoung Kim: juny924@korea.ac.kr

Highest degree: BS, Computer Science and Engineering, Hankuk University of Foreign Studies

Position title: MS. Candidate, Department of Industrial Management Engineering, Korea University

Areas of interest: Product Development, UX, UI

Jaeho Choe: jhchoe@daejin.ac.kr

Highest degree: $\mathrm{PhD}$, Department of Industrial Engineering, POSTECH

Position title: Professor, Department of Industrial Management

Engineering, Daejin University

Areas of interest: Product Development and Design

Eui S. Jung: ejung@korea.ac.kr

Highest degree: $\mathrm{PhD}$, Department of Industrial Engineering,

Pennsylvania State University

Position title: Professor, Department of Industrial Management Engineering, Korea University

Areas of interest: Product Development, Technology Management

Date Received : 2011-10-17

Date Revised :2011-12-01

Date Accepted : 2011-12-07 